\title{
Performance Comparison of Classification Methods with the Combinations of the Imputation and Gene Selection Methods
}

\author{
Donguk Kim ${ }^{1}$. Jin Hyun Nam ${ }^{2}$. Kyung Ha Hong ${ }^{3}$ \\ ${ }^{1}$ Department of Statistics, Sungkyunkwan University \\ ${ }^{2}$ Department of Statistics, Sungkyunkwan University \\ ${ }^{3}$ Korea Institute for Defense Analyses
}

(Received November 2011; accepted December 2011)

\begin{abstract}
Gene expression data is obtained through many stages of an experiment and errors produced during the process may cause missing values. Due to the distinctness of the data so called 'small $n$ large $p$ ', genes have to be selected for statistical analysis, like classification analysis. For this reason, imputation and gene selection are important in a microarray data analysis. In the literature, imputation, gene selection and classification analysis have been studied respectively. However, imputation, gene selection and classification analysis are sequential processing. For this aspect, we compare the performance of classification methods after imputation and gene selection methods are applied to microarray data. Numerical simulations are carried out to evaluate the classification methods that use various combinations of the imputation and gene selection methods.
\end{abstract}

Keywords: Gene expression, imputation, gene selection, classification.

\section{Introduction}

Microarray data typically have a relatively small sample size compared to the number of genes. Therefore, a process of selecting a useful gene is necessary to perform statistical analysis such as classification analysis or discriminant analysis. Also, missing values(MVs) of microarray data commonly occur during data preparation mainly due to imperfections in the various steps of microarray experiments. For this reason, MVs imputation and gene selection are important in microarray data analysis but MVs imputation, gene selection and classification analysis have been studied respectively (Kim et al., 2005; Scheel et al., 2005; Dudoit et al., 2002; Lee et al., 2005; Liew et al., 2010).

Since the classification analysis is generally incorporated with MVs imputation and gene selection, we compare the performance of classification methods using various combinations of the imputation and gene selection methods. Missing data set was made with missing rate of $1 \%, 4 \%, 7 \%, 10 \%$ and

${ }^{1}$ Corresponding author: Professor, Department of Statistics, Sungkyunkwan University, 53 MyeongnyunDong 3-Ga, Jongno-Gu, Seoul 110-745, Korea. E-mail: dkim@skku.edu 
$15 \%$ on each of three data sets and the process was repeated ten times respectively. To fill out MVs, we conducted four different imputation methods. For gene selection, four different methods were applied to each of the imputed data sets. Therefore, for three data sets, we applied five missing rates with ten repetitions at each missing rate, four imputation methods and four gene selection methods. Then seven classification methods were applied to 2,400 data sets to study the performance.

The paper is organized as follows. Section 2 briefly describes the recently developed four imputation, four gene selection and seven classification methods. In Section 3, we present the simulation setting and compare the performance of the classification methods with the combinations of several imputation and gene selection methods applied to microarray data. Finally, we summarize and discuss our major findings and make further suggestion.

\section{Methods}

In this section, we briefly summarize 4 imputation methods and 4 gene selection methods to make imputation data sets. Classification methods are then briefly explained.

\subsection{Imputation methods}

Given $n$ microarray experiments, each of experiments contains the expressions of $p$ genes, the data can be organized into an $n \times p$ matrix of gene expression values. Denote $x_{i j}$ to be the expression value of gene $j$ in the $i^{\text {th }}$ sample. We refer to a particular gene with MVs to be estimated as the target gene. The set of genes with available information to estimate the MVs of the target gene will be denoted as $C_{v}$, candidate genes.

$K$-nearest neighborhood(KNN) can be adapted to estimate continuous variables (Troyanskaya et al., 2001). To explain KNN, we first define the weighted Euclidean distance between target gene $\boldsymbol{x}_{j}$ and each candidate gene $\boldsymbol{x}_{k}, k \in C_{v}$ :

$$
d_{j k} \equiv d\left(\boldsymbol{x}_{j}, \boldsymbol{x}_{k}\right)=\left\{n_{j k}^{-1} \sum_{i=1}^{n} r_{i j} r_{i k}\left(x_{i j}-x_{i k}\right)^{2}\right\}^{\frac{1}{2}}, \quad k \in C_{v},
$$

where $n_{j k}=\sum_{i=1}^{n} r_{i j} r_{i k}$, and $r_{i j}=1$ if $x_{i j}$ is observed and $r_{i j}=0$ if $x_{i j}$ is missing. Then estimated $\mathrm{MV} \hat{y}_{i j}$ is as follows.

$$
\hat{y}_{i j}=\sum_{k \in C_{v}^{*}} w_{k} x_{i k}
$$

where $C_{v}^{*}$ is the set of the $K$ selected candidate genes, $w_{k}=1 /\left(d_{j k} C\right), k \in C_{v}^{*}$ is the weight and $C=\Sigma_{k \in C_{v}^{*}} d_{j k}^{-1}$ is the normalizing weight constant.

Instead of the weighted average of the $K$ available values, we can obtain an estimate of the MV of target gene $j$ based on repeated ordinary least squares(ROLS). The $k^{\text {th }}$ OLS imputation of a MV of a target gene $j$ based on available data is given by

$$
\hat{y}_{i j}^{(k)}=\bar{x}_{j}+b_{j}^{(k)}\left(x_{i k}-\bar{x}_{k}\right), \quad k \in C_{v}^{*},
$$

where $x_{i k}$ is the available value of candidate gene $k \in C_{v}^{*}, \bar{x}_{j}$ and $\bar{x}_{k}$ are the sample means based on jointly available data, and $b_{j}^{(k)}$ is the regression slope coefficient using the available data. Then 
estimated MV $\hat{y}_{i j}$ is as follows (Nguyen et al., 2004).

$$
\hat{y}_{i j}=\sum_{k \in C_{v}^{*}} w_{k} \hat{y}_{i j}^{(k)} .
$$

Partial least squares(PLS) is a dimension reduction method that extracts the gene components sequentially to maximize the sample covariance between the target gene and the linear combination of the set of candidate genes. The fitted expression values of target gene $j$ is $\hat{\boldsymbol{x}}_{j}=T(j) \hat{\boldsymbol{\beta}}_{j}$, where $T(j)$ is the matrix of the PLS gene components and $\hat{\boldsymbol{\beta}}_{j}$ is the least squares regression coefficient estimates. Then the estimates of the MVs of target gene $j$ is given by

$$
\hat{\boldsymbol{y}}_{j}=T^{*}(j) \hat{\boldsymbol{\beta}}_{j},
$$

where $T^{*}(j)$ is test PLS gene components (Nguyen et al., 2004).

The Bayesian principle component analysis(BPCA) method consists of principal component(PC) regression, Bayesian estimation and iterations based on expectation maximization(EM) algorithm. The fitted expression values of target gene $j$ is $\hat{\boldsymbol{x}}_{j}=T(j) \hat{\boldsymbol{\beta}}_{j}$, where $T(j)$ is the matrix of principal components scores and $\hat{\boldsymbol{\beta}}_{j}$ is the least squares regression coefficient estimates. Then the estimates of the MVs of target gene $j$ is computed as

$$
\hat{\boldsymbol{y}}_{j}=T^{*}(j) \hat{\boldsymbol{\beta}}_{j},
$$

where $T^{*}(j)$ are test principal components scores (Oba et al., 2003).

\subsection{Gene selection methods}

Four gene selection methods are introduced. These are BSS/WSS, soft thresholding, information gain and SVM-RFE criterion. Using these criterions, we select the gene that has the largest absolute value of the criterion and this process is continued until a certain number of genes are obtained.

Dudoit et al. (2002) suggested BSS/WSS criterion. For $K$ classes, the class labels $y_{i}$ 's are defined to be an integer ranging from 1 to $K$, and $n_{k}$ denotes the number of observations belong to class $k$. Then BSS/WSS is defined as

$$
\operatorname{BSS} / \operatorname{WSS}(j)=\frac{\sum_{i=1}^{n} \sum_{k=1}^{K} I\left(y_{i}=k\right)\left(\bar{x}_{k j}-\bar{x}_{j}\right)^{2}}{\sum_{i=1}^{n} \sum_{k=1}^{K} I\left(y_{i}=k\right)\left(x_{i j}-\bar{x}_{k j}\right)^{2}},
$$

where $\bar{x}_{j}$ denotes the average expression level of gene $j$ across all samples and $\bar{x}_{k j}$ denotes the average expression level of across samples belonging to class $k$ only.

Let $d_{k j}=\left(\bar{x}_{k j}-\bar{x}_{j}\right) /\left(m_{k} \cdot\left(s_{j}+s_{0}\right)\right)$, where $s_{j}^{2}=\Sigma_{k} \Sigma_{i \in C_{k}}\left(x_{i j}-\bar{x}_{k j}\right)^{2} /(n-K)$ is pooled within-class standard deviation for gene $j, C_{k}$ is $n_{k}$ samples in class $k, m_{k}=\sqrt{1 / n_{k}+1 / n}$ and $s_{0}$ is the median value of the $s_{j}, j=1, \ldots, p$ over the set of genes. Soft thresholding (Tibshirani et al., 2002) is defined by

$$
d_{k j}^{\prime}=\operatorname{sign}\left(d_{k j}\right)\left(\left|d_{k j}\right|-\Delta\right)_{+},
$$

where + means positive part, i.e, $t_{+}=t$ if $t>0$ and zero otherwise. 
The information gain(Info gain; $\operatorname{IG}\left(y, \boldsymbol{x}_{j}\right)$ ) of a given $\boldsymbol{x}_{j}$ with respect to the class $y$ is the reduction in uncertainty about the value of $y$ when we know the value of $\boldsymbol{x}_{j}$ (Liu et al., 2005). When the entropy $H(y)$ is defined as $H(y)=-\Sigma_{k=1}^{K} P(y=k) \log P(y=k)$, the information gain is as follows.

$$
\mathrm{IG}\left(y, \boldsymbol{x}_{j}\right)=H(y)-H\left(y \mid \boldsymbol{x}_{j}\right) .
$$

Support vector machines-recursive feature elimination(SVM-RFE) generates the ranking of genes using backward elimination. The main idea of SVM-RFE is the selection of a gene with largest weight. Thus, an iterative procedure trains the SVM classifier, computes the ranking $w_{j}^{2}$ for all genes that minimize $\|\boldsymbol{w}\|^{2} / 2+C \sum_{i=1}^{n} \xi_{i}$ subject to $y_{i}\left(\boldsymbol{w} \cdot \boldsymbol{x}_{i}+b\right) \geq 1-\xi_{i}, \xi_{i} \geq 0$, where $\xi_{i}$ is a slack variable and then removes the gene with the smallest ranking criterion. The procedure is iterated until a certain number of selected genes is obtained (Guyon et al., 2002).

\subsection{Classification methods}

We briefly explains seven classification methods. Fisher linear discriminant analysis(FLDA) projects high-dimensional data onto a line and performs classification in this one-dimensional space. FLDA is based on finding linear combinations $\boldsymbol{x}^{\prime} \boldsymbol{a}$ which maximizes $\boldsymbol{a}^{\prime} B \boldsymbol{a} / \boldsymbol{a}^{\prime} W \boldsymbol{a}$, where $B$ and $W$ denote matrices of between-group and within-group sums of squares, respectively.

For multivariate normal class densities, the maximum likelihood discriminant rule is $\operatorname{argmin}_{k}\{(\boldsymbol{x}-$ $\left.\left.\mu_{k}\right)^{\prime} \Sigma_{k}^{-1}\left(\boldsymbol{x}-\mu_{k}\right)+\log \left|\Sigma_{k}\right|\right\}$. Linear discriminant analysis(LDA) assumes that the class densities have the same covariance matrix, $\Sigma_{k}=\Sigma$, and the diagonal linear discriminant analysis(DLDA) assumes that the class densities have the same diagonal covariance matrix $\Sigma_{k}=\operatorname{diag}\left(\sigma_{1}^{2}, \ldots, \sigma_{p}^{2}\right)$.

$K$-nearest neighborhood(KNN) is a method that classifies unlabeled samples based on their similarity with samples in the training set. KNN finds the $K$ closest samples in the training set and assigns to the class that appears most frequently within the $K$ subsets. Classification and regression tree(CART) is a tree-building technique. CART builds the tree using the recursive splitting of nodes and assigns it to the class that appears most frequently within terminal node.

SVM separates the data into different classes using a hyper-plane $\boldsymbol{w} \cdot \boldsymbol{x}+b=0$ corresponding to the decision function $f(\boldsymbol{x})=\operatorname{sign}(\boldsymbol{w} \cdot \boldsymbol{x}+b)$ which minimize $\|\boldsymbol{w}\|^{2} / 2+C \Sigma_{i=1}^{n} \xi_{i}$ subject to $y_{i}\left(\boldsymbol{w} \cdot \boldsymbol{x}_{i}+b\right) \geq 1-\xi_{i}, \xi_{i} \geq 0$, where $\xi_{i}$ is a slack variable.

Lastly, Naïve Bayes classifier(NB) is a simple probabilistic classifier based on the application of the Bayes theorem with naïve independence assumptions. The probability of class $k$ given $\boldsymbol{x}$ is

$$
P(y=k \mid X=\boldsymbol{x})=\prod_{j=1}^{p} \frac{f\left(x_{j} \mid y=k\right) \cdot P(y=k)}{C}
$$

where $C$ is a constant scaling factor based on the genes $x_{j}, j=1, \ldots, p$, and $y$ is the class variable. NB assigns the class of $\boldsymbol{x}$ to $k^{*}$ if $k^{*}=\operatorname{argmax}_{k} P(y=k \mid X=\boldsymbol{x})$.

\section{Performance Comparison}

To compare the performance of classification methods using the various combinations of the imputation and gene selection methods, we generated a missing data set at the missing rate of $1 \%$, $4 \%, 7 \%, 10 \%$ and $15 \%$ from each original data set. This missing data set was generated ten times respectively at each missing rate. An imputed data set was obtained after the application of 4 imputation methods to the missing data set. 


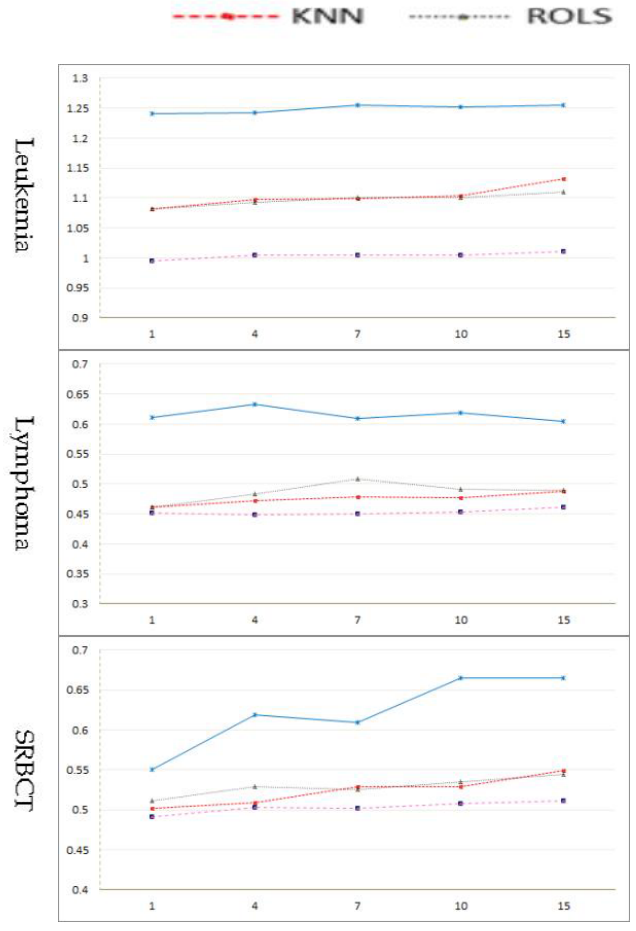

(a) RMSE
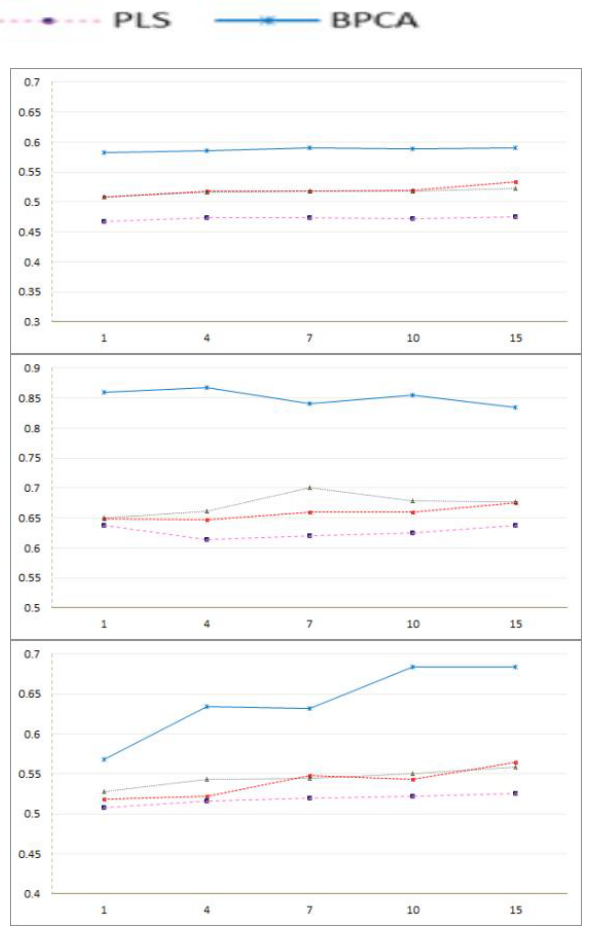

(b) NRMSE

Figure 3.1. Comparison of RMSE and NRMSE

\subsection{Dataset}

In this study, we used three popular microarray data sets. The Leukemia data set have 3,032 genes and 72 samples (Golub et al., 1999). The data set was obtained after three pre-processing described in Dudoit et al. (2002). The Lymphoma data set have 4,026 genes and 62 samples (Alizadeh et al., 2000). The Small round blue cell tumor(SRBCT) data set have 2,308 genes and 63 samples (Khan et al., 2001).

\subsection{Comparison of RMSE and NRMSE}

We used root mean square error(RMSE) and normalized root mean square error(NRMSE) for the evaluation of MVs imputation. RMSE and NRMSE are a frequently used measure of the differences between values predicted by a model or an estimator and the values actually observed. Let $y$ be the true expression values, $\hat{y}$ be its estimated values and $M$ be the number of missing values. RMSE and NRMSE are defined as

$$
\begin{aligned}
\operatorname{RMSE} & =\sqrt{\sum_{i=1}^{M} \frac{\left(y_{i}-\hat{y}_{i}\right)^{2}}{M}} \\
\mathrm{NRMSE} & =\operatorname{RMSE} / \operatorname{std}(y),
\end{aligned}
$$

where std is standard deviation. 


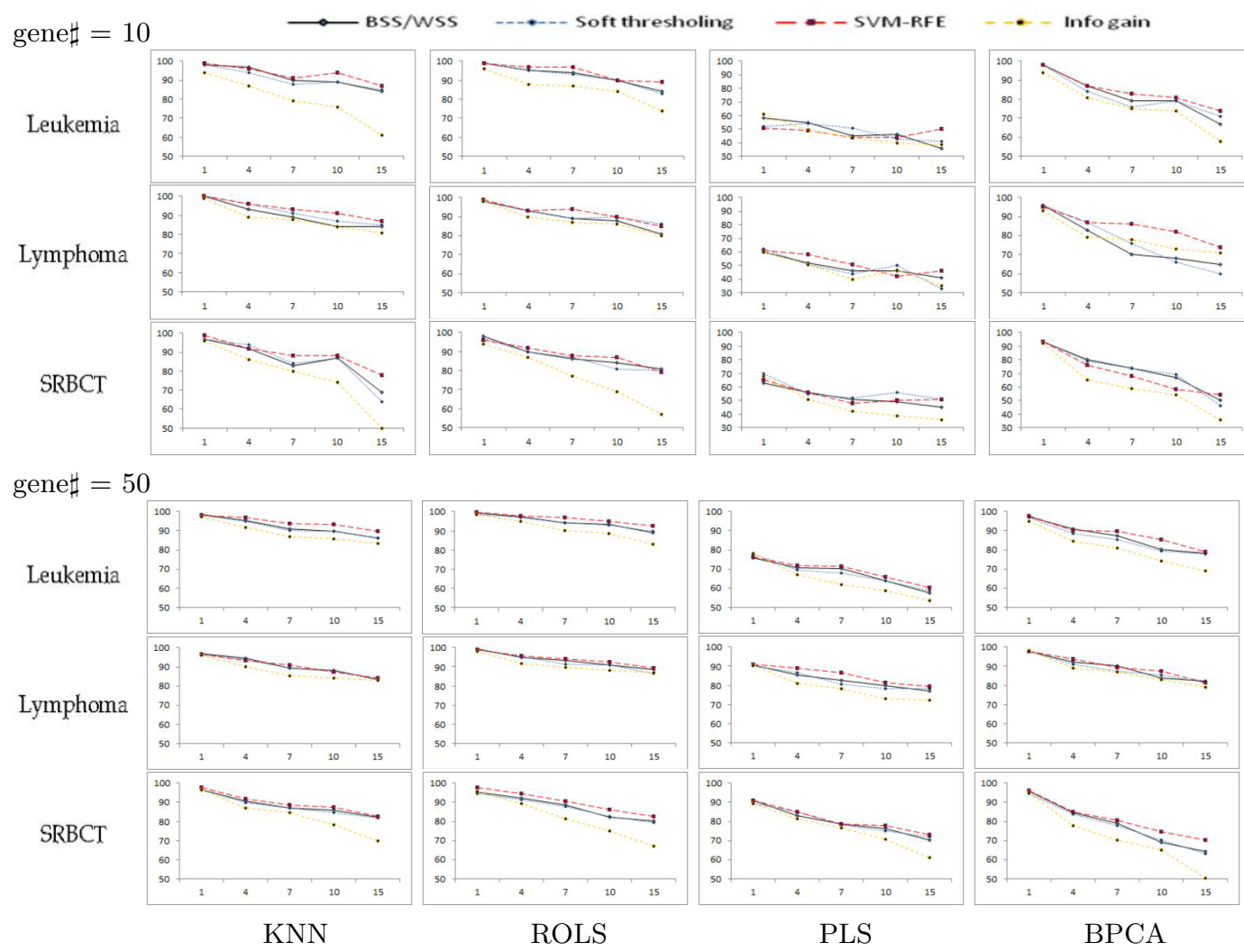

Figure 3.2. Comparison of gene selection rate after imputation

Figure 3.1 shows the performance of four imputation methods, KNN, ROLS, PLS and BPCA methods corresponding to missing rate. In terms of RMSE and NRMSE, it was observed that the PLS method showed the best performance whereas the BPCA method showed the worst performance. The performance of KNN and ROLS was intermediate between PLS and BPCA. These trends were consistent regardless of the missing rate for all three data sets. These four types of imputation methods seem to differ in terms of their performance, however, the difference was small.

\subsection{Comparison of gene selection rate after imputation}

In Figure 3.2, matched gene rate between original data set and imputed data set is compared after four gene selection methods are applied to the imputed data set and original data set. The $X$ and $Y$ axes stand for the missing rate and the percentage of identically selected genes, respectively when the four gene selection methods are applied to the original data set and imputed data set.

When the number of the selected gene is 10, for example, BSS/WSS, soft thresholding and SVMRFE methods showed a high level of matching between the genes selected from the original data set and those from the imputed data set. In the case of the SVM-RFE method, generally, the matching rate of the genes selected from two data sets was observed to be relatively higher than other methods. 
Table 3.1. Misclassification rate of the original dataset

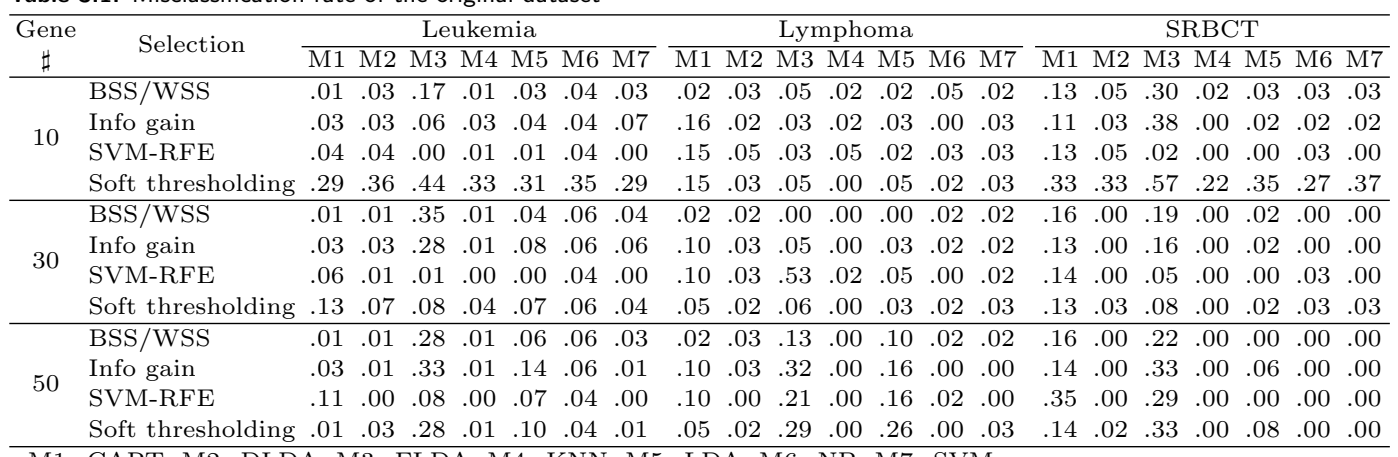

M1: CART, M2: DLDA, M3: FLDA, M4: KNN, M5: LDA, M6: NB, M7: SVM

When considering missing rate, the matching rate declines relatively little even with an increased missing rate for BSS/WSS, soft thresholding and SVM-RFE methods; however, an increased missing rate resulted in relatively sharp decline of the matching rate for the Info gain. When considering imputation methods, KNN and ROLS provided higher matching rates; however, PLS showed a relatively lower matching rate, while the BPCA provided a sharp decline of the matching rate when the missing rates were increased.

The results did not differ much when the number of genes is 50. For summary, BSS/WSS, soft thresholding and SVM-RFE showed a relatively small decline of matching rate while SVM-RFE showed a higher matching rate of genes. SVM-RFE can be recommended for the gene selection method, because it showed robust results irrespective of the missing rates and imputation methods.

\subsection{Misclassification rate of imputed data set}

To compare the performance of the classification methods, we used the mean of misclassification rates and the difference between the misclassification rate of the original data set and the average misclassification rate of the imputed data sets for the combination of four imputation methods and four gene selection methods. Table 3.1 displays the misclassification rate of original data set with four gene selection methods when the number of selected gene is 10, 30 and 50, respectively.

In Figure 3.3 to Figure 3.5, the $X$-axis represents a combination of the imputation methods and gene selection methods. Each point denotes following combination:
1: $\mathrm{BPCA} \times \mathrm{BSS} / \mathrm{WSS}$
2: BPCA $\times$ Info gain
3: BPCA $\times$ SVM-RFE
4: BPCA $\times$ Soft thresholding
5: $\mathrm{KNN} \times \mathrm{BSS} / \mathrm{WSS}$
6: KNN $\times$ Info gain
7: $\mathrm{KNN} \times \mathrm{SVM}-\mathrm{RFE}$
8: $\mathrm{KNN} \times$ Soft thresholding
9: $\mathrm{PLS} \times \mathrm{BSS} / \mathrm{WSS}$
10: $\mathrm{PLS} \times$ Info gain
11: $\mathrm{PLS} \times \mathrm{SVM}-\mathrm{RFE}$
12: PLS $\times$ Soft thresholding
13: $\mathrm{ROLS} \times \mathrm{BSS} / \mathrm{WSS}$
14: ROLS $\times$ Info gain
15: ROLS $\times$ SVM-RFE
16: ROLS $\times$ Soft thresholding

The Figure 3.3(a), Figure 3.4(a) and Figure 3.5(a) show the average of 5 misclassification rates and Figure 3.3(b), Figure 3.4(b) and Figure 3.5(b) show the difference between the misclassification rate of original data set and the average misclassification rate of the imputed data sets. For the classification analysis in the microarray data, the performance can be considered good and robust when the misclassification rate is low and the difference between two misclassification rates from the original data set and the imputed data set is small.

Figure 3.3(a) and Figure 3.3(b) are the results from the Leukemia data set. When the number of 

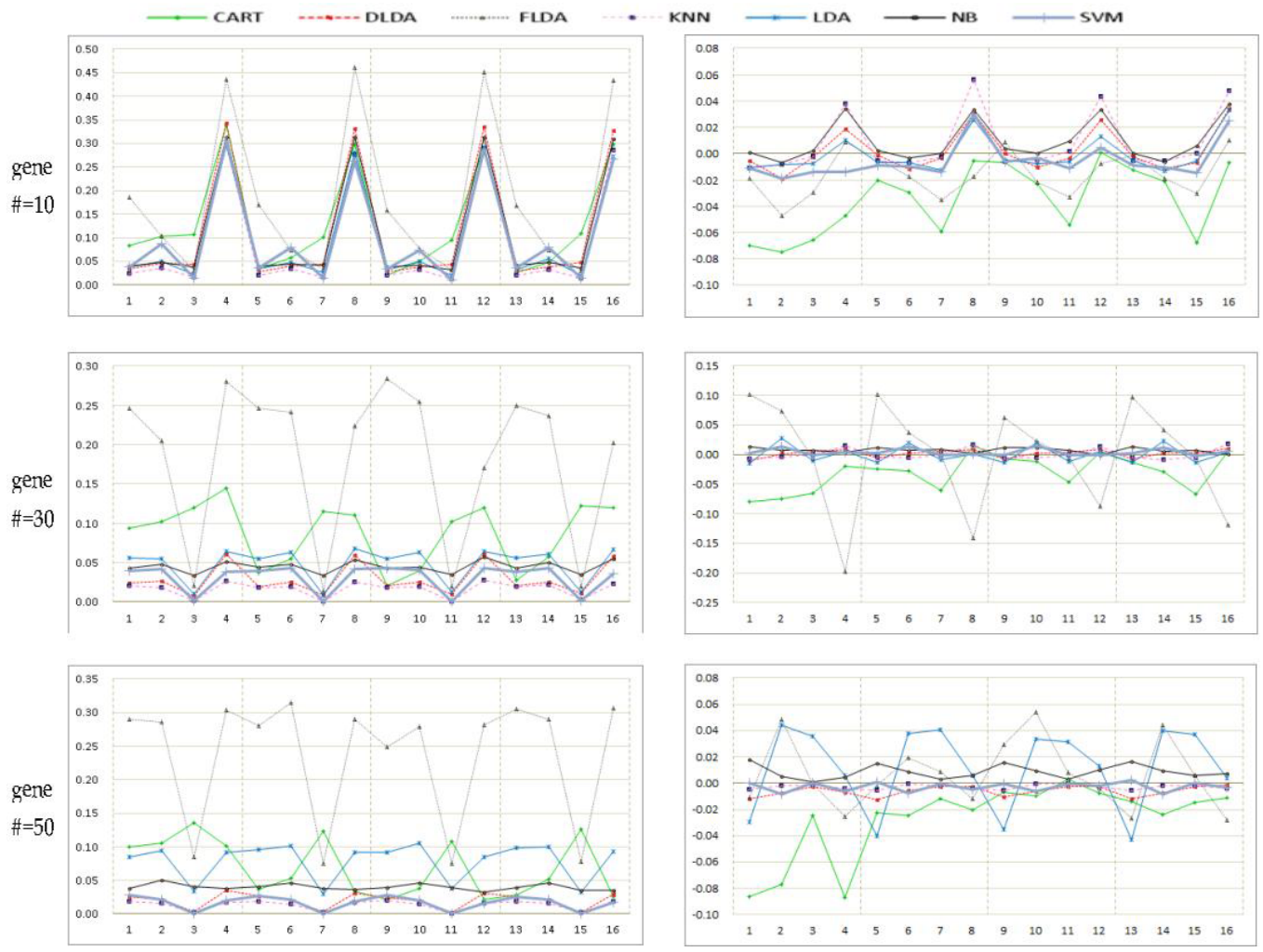

(a) Mean of 5 misclassification rates of Leukemia

(b) Difference between misclassification rates of Figure 3.3 Leukemia

genes is 30, DLDA, KNN and SVM methods showed relatively low misclassification rates compared to FLDA, CART, LDA and NB from the imputed data sets. Similar results were observed for the difference of misclassification rates.

DLDA, KNN and SVM methods showed especially good performance when SVM-RFE was used for the gene selection method irrespective of the imputation methods. This result was consistent regardless of the number of genes. Figure 3.4(a) and Figure 3.4(b) are the results from Lymphoma data set. When the number of genes is 30, DLDA, KNN, NB and SVM methods showed relatively low misclassification rates compared to FLDA, CART and LDA, and similar results were observed for the difference of misclassification rates. Figure 3.5(a) and Figure 3.5(b) are the results from the SRBCT data set. When the number of genes is 30, DLDA, KNN, LDA, NB and SVM methods showed relatively low misclassification rates compared to FLDA and CART. We have similar results for the difference of misclassification rates. For Leukemia, Lymphoma and SRBCT data set, SVMRFE was recommended for the gene selection method when we compared misclassification rates. In summary, among all classification methods, DLDA, KNN and SVM methods showed better performance in terms of misclassification rate when compared to FLDA, CART, LDA and NB methods. When considering various combinations of the imputation and gene selection methods (irrespective of the imputation method) SVM-RFE method provides good performance for the 

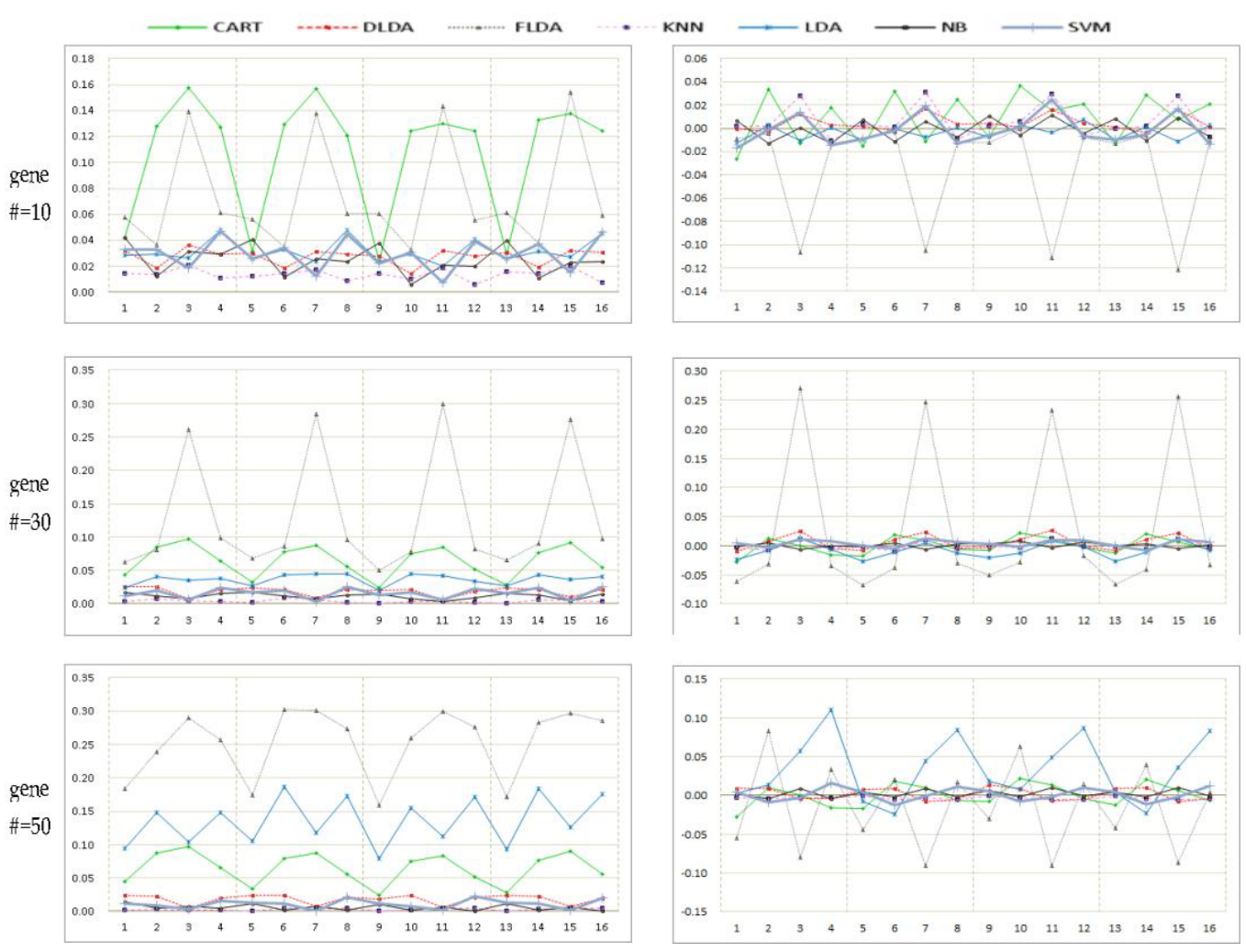

(a) Mean of 5 misclassification rates of Lymphoma

(b) Difference between misclassification rates of Lymphoma

Figure 3.4

misclassification rate and the difference of misclassification rates of original data set and imputed data sets. This result was consistent regardless of the number of genes and data sets.

\section{Discussion}

Microarray data is obtained through many stages of experiment and errors produced during the process may cause missing values. Due to the distinctness of the data, so called 'small $n$ large $p$ ', genes have to be selected for statistical analysis. Thus, preprocessing (such as missing values substitution and gene selection) is indispensable. Such preprocessing influence a series of follow-up analysis results.

The result of gene selection is affected by an imputation method as well as a gene selection method when there is a missing value in original data set. That is, the imputed data set obtained by the imputation method might have some change in data characteristics, hence it affects the result of gene selection. Furthermore, different gene selection methods will make different data sets and might influence the result of the classification method.

PLS showed good performance in RMSE aspect. However, the PLS imputation method was un- 

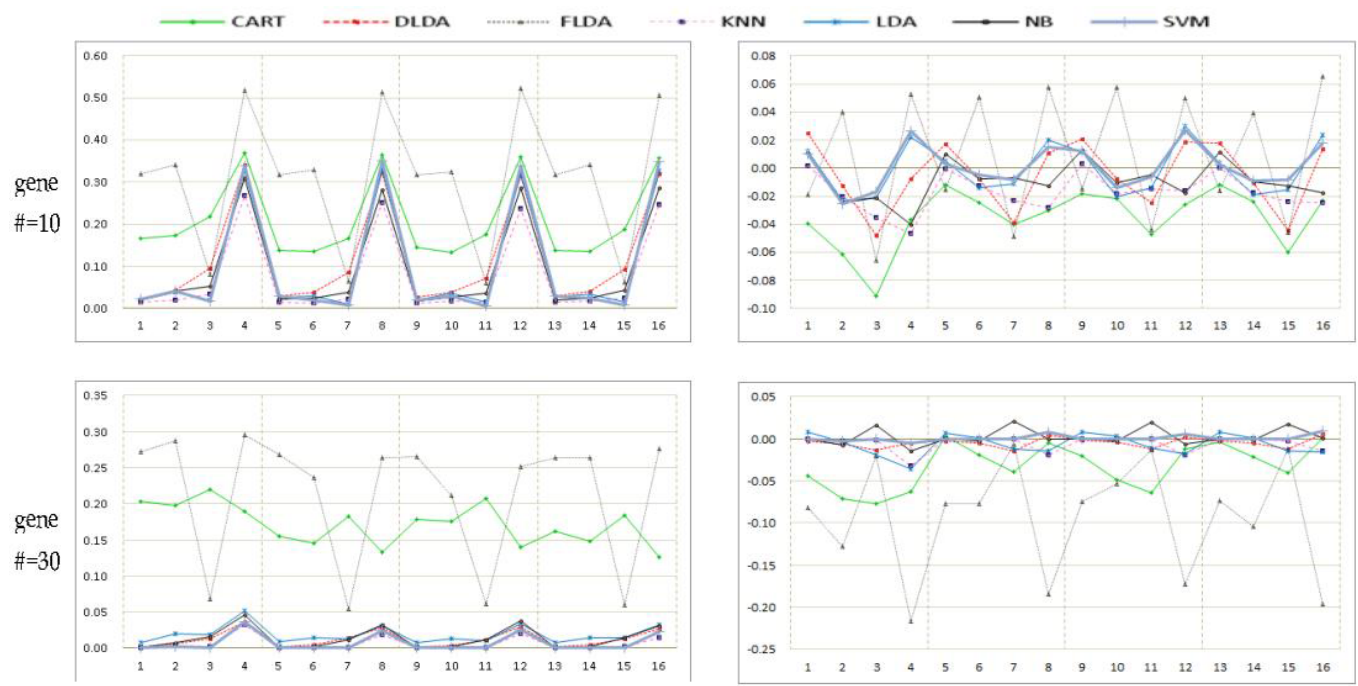

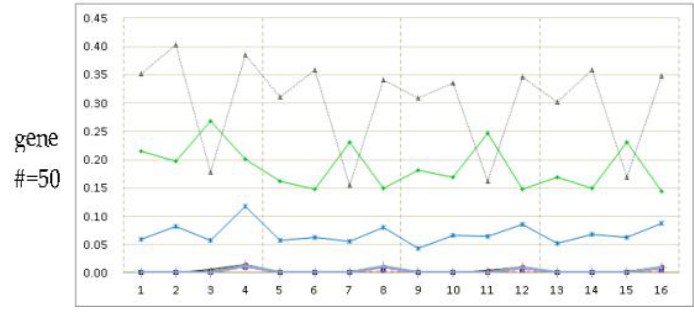

(a) Mean of 5 misclassification rates of SRBCT

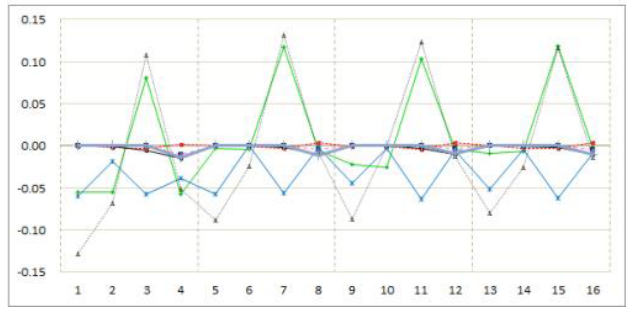

(b) Difference between misclassification rates of SRBCT

Figure 3.5

advisable when the gene selection rate was compared. In the RMSE aspect, the performance of KNN and ROLS methods were not bad; in addition, the matching rate of genes were observed to be high if combined with gene selection methods. In addition, when KNN and ROLS methods were combined with BSS/WSS, Soft thresholding and SVM-RFE methods, good performance was observed. Particularly, SVM-RFE had the best performance among the methods. Lastly, our goal was to compare the performance of classification methods after applying preprocessing methods instead of the gene selection method itself; hence SVM-RFE might be recommended in the gene selection method.

For studying the performance of classification methods, we computed the misclassification rate of the original data set and imputed data set that was made through the imputation and gene selection methods. First, in the point of low misclassification rate from the imputed data set i) for Leukemia, the misclassification rate was between $0.03 \sim 0.05$ in the order of KNN, SVM and DLDA that indicated relatively better performance than other methods; ii) for Lymphoma, the misclassification rate was between $0.01 \sim 0.02$ in the order of KNN, NB, SVM and DLDA; iii) for SRBCT, the misclassification rate was between $0.02 \sim 0.03$ in the order of SVM, KNN and NB.

Second, for the difference between misclassification rates in the original and imputed data sets, 
the difference range was i) $-0.01 \sim 0.01$ for DLDA, KNN, LDA, NB and SVM for Leukemia; ii) $0.00 \sim 0.01$ for DLDA, KNN, NB and SVM for Lymphoma; and iii) $-0.02 \sim 0.01$ for DLDA, KNN, $\mathrm{NB}$ and SVM for SRBCT.

In summary, DLDA, KNN and SVM methods are stable and robust irrespective of the gene selection methods or imputation methods and also give a low misclassification rate. Thus, DLDA, KNN and SVM methods are recommended for discriminant analysis that uses the microarray data with missing values.

\section{References}

Alizadeh, A. A., Eisen, M. B., Davis, R. E., Ma, C., Lossos, I. S., Rosenwald, A., Bordrick, J. C., Sabet, H., Tran, T., Yu, X., Powell, J. I., Yang, L., Marti, G. E., Moore, T. Jr. J. H., Lu, L., Lwis, D. B., Tibshirani, R., Sherlock, G., Chan, W. C., Greiner, T. C., Weisenburger, D. D., Armitage, J. O., Warnke, R., Levy, R., Wilson, W., Grever, M. R., Byrd, J. C., Botstein, D., Brouwn, P. O. and Staudt, L. M. (2000). Distinct types of diffuse large B-cell lymphoma identified by gene expression profiling, Nature, 403, 503-511.

Dudoit, S., Fridlyand, J. and Speed, T. P. (2002). Comparison of discrimination methods for the classification of tumors using gene expression data, Journal of the American Statistical Association, 97, 77-87.

Golub, T. R., Slonim, D. K., Tamayo, P., Huard, C., Gaasenbeek, M., Mesirov, K. P., Coller, H., Loh, M., Downing, J. R., Caligiuri, M. A., Bloomfield, C. D. and Lander, E. S. (1999). Molecular classification of cancer: Class discovery and class prediction by gene expression monitoring, Science, 286, 531-537.

Guyon, I., Weston, J. and Barnhill, S. (2002). Gene selection for cancer classification using support vector machines, Machine Learning, 46, 389-422.

Khan, J., Wei, J., Ringner, M., Saal, L., Ladanyi, M., Westermann, F., Berthold, F., Schwab, M., Antonescu, C., Peterson, C. and Meltzer, P. S. (2001). Classification and diagnostic prediction of cancers using gene expression profiling and artificial neural networks, Nature Medicine, 7, 673-679.

Kim, H., Golub, G. H. and Park, H. (2005). Missing value estimation for DNA microarray gene expression data: Local least squares imputation, Bioinformatics, 21, 187-198.

Lee, J. W., Lee, J. B., Park, M. and Song, S. H. (2005). An extensive comparison of recent classification tools applied to microarray data, Computational Statistics and Data Analysis, 48, 869-885.

Liew, A. W., Law, N. and Yan, H. (2010). Missing value imputation for gene expression data: computational techniques to recover missing data from available information, Briefings in Bioinformatics, 12, 498513.

Liu, X., Krishnan, A. and Mondry, A. (2005). An entropy-based gene selection method for cancer classification using microarray data, BMC Bioinformatics, 6, 76.

Nguyen, D. V., Wang, N. and Carroll, R. J. (2004). Evaluation of missing value estimation for microarray data, Journal of Data Science, 2, 347-370.

Oba, S., Sato, M., Takemasa, I., Monden, M., Matsubara, K. and Ishii, S. (2003). A Bayesian missing value estimation method for gene expression profile data, Bioinformatics, 19, 2088-2096.

Scheel, I., Aldrin, M., Glad, I. K., Sorum, R., Lyng, H. and Frigessi, A. (2005). The influence of missing value imputation on detection of differentially expressed genes from microarray data, Bioinformatics, 21, $4272-4279$

Tibshirani, R., Hastie, T., Narasimhan, B. and Chu, G. (2002). Diagnosis of multiple cancer types by shrunken centroids of gene expression, Proceedings of the National Academy of Sciences, 99, 6567-6572.

Troyanskaya, O., Cantor, M., Sherlock, G., Brown, P., Hastie, T., Tibshirani, R., Botstein, D. and Altman, R. B. (2001). Missing value estimation methods for DNA microarrays, Bioinformatics, 17, 520-525. 